\title{
PHYTOCHEMICAL ANALYSIS AND ANTIFUNGAL ACTIVITY OF GYMNOSPERM AGAINST FUSARIUM WILT OF BANANA
}

\author{
AIN, Q. U. ${ }^{1 *}-$ ASAD S. $^{2}-$ JAMAL, A. ${ }^{2}-$ ARIF, M. ${ }^{3}-$ MAHMOOD-UL-HASSAN, M. ${ }^{4}$ \\ ${ }^{l}$ PARC Institute of Advanced Studies in Agriculture, National Agricultural Research Center, \\ Islamabad, Pakistan
}

${ }^{2}$ Crop Diseases Research Institute, National Agricultural Research Centre, Islamabad, Pakistan

${ }^{3}$ Plant Pathology Department, The University of Agriculture, Peshawar, Pakistan

${ }^{4}$ Land Resources Research Institute, National Agricultural Research Centre, Islamabad, Pakistan

${ }^{*}$ Corresponding author

e-mail: quratulainsatti@yahoo.com; phone: +92-344-810-7441

(Received 22 $2^{\text {nd }}$ Sep 2020; accepted $9^{\text {th }}$ Apr 2021)

\begin{abstract}
Fusarium vascular wilt caused by hyphomycete i.e. Fusarium oxysporum f. sp. cubense (Foc) exterminated the banana industry of Central America due to its dependence on monoculture. In this investigation, six gymnosperm plants i.e. Cupressus sempervirens, Thuja orientalis, Cedrus deodara, Pinus wallachiana, Picea smithiana and Pinus roxburghii were evaluated for their ability to control Foc; Tropical race 4 (TR4). Leaf and cone extracts (5-10\%) of all six plants were screened using poisoned food technique. Extracts recording higher percent inhibition $(\geq 60 \%)$ were further evaluated, an in vitro and a pot experiment. Highest percent inhibition in the in vitro assay and lowest disease severity index (DSI) in the pot experiment were recorded for ethanol leaf extract of $P$. wallachiana $\left(\mathrm{Pw}_{\mathrm{EL}}\right)$, followed by $T$. orientalis ethanol leaf extract (ToEL). The Gas Chromatography-Mass Spectrometry (GC-MS) analysis of both extracts was performed and the results indicated detection of seven compounds. Three of the compounds viz cis-9-Hexadecenal, 1-Naphthalenepentanoic acid and 1-Naphthalenecarboxylic acid were found in both extracts whereas Vitamin C, Hexatriacontyl trifluoroacetate, Oleic acid, delta-Cadinene were found in P $\mathrm{w}_{\mathrm{EL}}$ while Cedrol, Podocarp-7-en-3-one, 1-Phenanthrenecarboxylic acid and Pimaric acid were identified in ToEL. It is hypothesized that these phytocompounds might be the possible reason of Foc inhibition in current studies.
\end{abstract}

Keywords: Fusarium oxysporum $f$. sp. cubense, poisoned food technique, disease severity index, Gas Chromatography-Mass Spectrometry, phytocompounds

\section{Introduction}

Banana belongs to Musa genus and Asia is its center of origin (Ploetz et al., 2007). Trade quality banana are parthenocarpic and triploids that are vegetatively propagated (Simmonds, 1986). Even though hundreds of banana cultivars exist globally, majority of the production is based on few cultivars (Perrier et al., 2011). Panama epidemic devastated banana industry of Central America that was exclusively growing susceptible variety Gros Michel (Drenth and Guest, 2016). There was a major substitution of susceptible banana with resistant Cavendish cultivars (Ghag et al., 2015). Due to dissemination of diseased rhizomes and suckers intercontinental spread of the fusarium wilt occurred. Fusarium wilt is reported in most of the banana growing regions of the world e.g. Australia, Asia, Tropical Americas and Africa. The disease is induced by a hyphomycete named Fusarium oxysporum f. sp. cubense (Ploetz, 2000, 2006). External disease symptoms consist of leaf yellowing of older leaves, pseudostem splitting followed 
by leaf buckling and wilting (Yin et al., 2011). Internal symptoms consist of vascular discoloration blocking water conducting xylem vessels (Mackesy and Sullivan, 2015).

There are four recognized races of Foc separated on the basis of host susceptibility. Race 4 attacks Cavendish cultivars as well as those varieties that are attacked by race 1 and 2. Race 4 has two recognized strains i.e. TR4 (tropical race 4) and SR4 (subtropical race 4). The SR4 attacks banana that are growing suboptimally while TR4 attack Cavendish banana under any growing conditions (Ploetz, 2015; Drenth and Guest, 2016). Intercontinental spread of infected rhizome bits and suckers has led to the transmission of Foc TR4 from Taiwan to China, Indonesia, Philippines, Malaysia, Myanmar, Australia, Laos, India, Pakistan, Oman, Lebanon, Israel, Jordan and Mozambique. Dissemination of TR4 is much quicker (Drenth and Guest, 2016; Dita et al., 2018).

In Pakistan Fusarium wilt was reported in small farm, Thattha district in 2012-13 (Syed et al., 2015). Pakistan banana industry upto $95 \%$ of the area is based on Dwarf Cavendish (Basrai) cultivation. Presence of Foc TR4 is an alarming situation that if not controlled could lead to devastation as observed in past. Cultural practices i.e. flood-fallowing, suppressive soil, soil solarization and crop rotation are proposed (Stover, 1962) but have limited control (Gnanasekaran et al., 2015; Pegg et al., 2019). Development of resistant cultivars from diploids that are seed bearing is very expensive and time consuming (Ortiz and Swennen, 2014).

Different control options have been seeked in the recent decades for Foc management using chemicals, antagonistic microbes and botanicals against Foc. These management studies reported that control comes either by directly affecting the Foc morphology or by stimulating the host response. That is achieved from chemical compounds that might be the fungicide or the metabolites of plants and microbes. Many research investigations comprising on the efficacy of botanicals indicated that the secondary metabolites of higher plants possess such phytochemicals that had antimicrobial activity against phytopathogens (Doughari et al., 2009; Saravanakumar et al., 2015). Gymnosperms have never been tested against Foc although their antimicrobial activity is well known. This study addressed wilt problem using gymnosperm botanicals as control option for substituting conventional fungicide use. Six gymnosperm plants were selected including Cupressus sempervirens, Cedrus deodara, Picea smithiana, Pinus wallachiana, Pinus roxburghii and Thuja orientalis.

\section{Materials and methods}

\section{Acquisition, revival and confirmation of fungal culture}

Fusarium oxysporum f. sp. cubense (Foc; TR4) was graciously provided by the Tissue culture lab at National Agricultural Research Centre (NARC), Islamabad. Its molecular confirmation tests had already been performed by tissue culture department that isolated Foc from the diseased banana rhizomes collected from Thatta district of Sindh, Pakistan (Muhammad et al., 2017). The Foc culture was revived on Potato Dextrose Agar (PDA) and its morphological characters were analysed. Microscopic studies showed typical conidial morphology of Foc TR4. Microconidia are kidney shaped, hyaline, produced on false heads, mostly without septation. Macroconidia were sickle-shaped, hyaline, pointed at both ends, mostly 3-5 septate, borne on single phialides. 


\section{Collection and drying of samples}

Fresh leaves and cones of selected botanicals were collected from Murree $\left(33^{\circ} 54^{\prime} 15^{\prime \prime} \mathrm{N}\right.$ $73^{\circ} 23^{\prime} 25^{\prime \prime} \mathrm{E}$ with $2291 \mathrm{~m}$ altitude) and Swat $\left(35^{\circ} 12^{\prime} \mathrm{N} 72^{\circ} 29^{\prime} \mathrm{E}\right.$ with $980 \mathrm{~m}$ altitude) in the year 2014 and brought to fungal pathology lab at Crop Diseases Research Institute (CDRI), NARC, Islamabad, Pakistan. The samples were thoroughly washed and disinfected with 5\% Clorox. Collected samples were kept under shade for one month. Dried samples (leaf and cone) were ground in an electric grinder and stored in labeled air tight containers till further use.

\section{Extraction of samples}

The n-hexane, ethanol and methanol were used as solvents for extraction. Two concentrations (5\% and 10\%) for each sample were prepared (Monteiro et al., 2013). Powdered samples were individually mixed with $100 \mathrm{~mL}$ solvent in flasks (Erlenmeyer) and shaken at $60 \mathrm{rpm}$ (revolution per minute) for 48 hours. Extract in each flask was filtered and filtrate's solvent was removed by rotary evaporator (Sati and Joshi, 2010). These extracts were enclosed in labeled vials (Bajpai and Kang, 2010).

\section{Evaluation of antifungal potential of the plant extracts}

The evaluation of the antifungal potential of solvent extracts was done using food poisoning technique (Nene and Thapilyal, 2000; Monteiro et al., 2013). Each solvent extracts were amended with autoclaved PDA media and then poured into sterilized 90-mm petri plates. Disk of Foc $(6 \mathrm{~mm})$ was placed aseptically in the center of each poisoned petri plate. Plates having PDA with only $5 \%$ solvent in it, served as the control. Each treatment comprised of five replicates and plates were incubated $\left(25 \pm 2{ }^{\circ} \mathrm{C}\right)$. Radial mycelia growth (RMG) of Foc was recorded from second day after inoculation till that day on which plates of control treatment were completely filled with mycelial growth of Foc. For each treatment, percent inhibition was calculated by using formula:

$$
\text { Percent Inhibition }=\frac{\text { RMG of Control }- \text { RMG of Treatment }}{\text { RMG of Control } \times 100}
$$

\section{The in vitro assay and pot experiment of selected solvent extracts}

Selected solvent extracts were further investigated in the in vitro assay using $50 \%$ concentration to determine the most effective botanical. Only 5\% solvents amended with PDA, served as a solvent control. The PDA plates having only Foc disk, without any solvent and extract, served as positive control whereas the Propiconazole fungicide (Tilt) served as negative control $(100 \mu \mathrm{g} / \mathrm{mL})$. Assay was performed in the same manner as described earlier and percent inhibition of each treatment was calculated.

For pot experiment, four months old tissue cultured banana plants belonging to Dwarf Cavendish were grown in pots $(20 \mathrm{~cm} \times 25 \mathrm{~cm})$ with peat moss and soil in 2:1 ratios were used as potting mix and freshly grown five day old Foc TR4 culture was used for inoculation. The inoculation of Foc was done using soil impregnation with spore suspension at $10^{6}$ conc. (Huang et al., 2012). Four type of treatments were used in the pot assay viz positive control, solvent controls, negative control and solvent extracts

1) Simple control (positive control): Banana plants without any treatment. 
2) Solvent controls: n-hexane, ethanol and methanol (5\% conc.) without any plant extract.

3) Fungicide treatment (negative control): Propiconazole (Tilt $=100 \mu \mathrm{g} / \mathrm{mL}$ ) was used as fungicide.

4) Botanical treatments: $50 \%$ conc. of selected solvent extract.

The banana plant roots were first dipped in the treatment (botanicals/ 5\% respective solvents/ fungicide) for 35 minutes and were then sown in the potting mixture of the pots already impregnated with fungal spore suspension. Three replications for each treatment were used. Scale of external symptoms (Vicente et al., 2014) for Fusarium wilt was used for disease evaluation.

For each treatment, DSI was calculated (Huang et al., 2012) using formula:

$$
\text { DSI }=\frac{\sum(\text { Class } \times \text { No.of plants in that class })}{\text { Total no.of assessed plants } \times 5} \times 100
$$

When the clear disease symptoms appeared on the banana plants after inoculation, visual wilt symptom assessment was done after every 2 months. Plant growth parameters i.e. leaf length, pseudostem length and leaf width were also recorded on termination of pot experiment.

\section{Statistical analysis of experimental data}

Experimental results were analyzed using the Statistix (ver. 8.1.) software. The ANOVA (analysis of variance) was performed for each experiment and the results were compared through Least Significant Difference (LSD) between means at $p<0.05$.

\section{Gas Chromatography and Mass Spectrometry (GC-MS)}

Botanicals were prepared with $100 \mathrm{mg} / \mathrm{mL}$ in $100 \%$ pure ethanol and $0.45 \mu \mathrm{m}$ membrane filter was used to filter the botanicals. GC-MS analysis was done according to Karpagasundari and Kulothungan (2014) methodology with slight modifications. The GC-MS analysis of the most effective botanicals was executed using Shimadzu (GCMSQP2010 Ultra) comprising an auto sampler (AOC-20i) and gas chromatograph that is interfaced to mass spectrometer (MS) instrument employed with as following specifications:

$\begin{array}{ll}\text { Column } & \text { DB 5 Ms }(30 \mathrm{~m} \times 0.25 \mathrm{~mm} \times 0.25 \mu \mathrm{m}) \\ \text { Injection volume } & 3 \mu \mathrm{L}(10: 1 \text { split ratio }) \\ \text { Ion-source } & 200^{\circ} \mathrm{C}\end{array}$

temperature

Carrier gas

Injector temperature

Helium $(99.99 \%)$ at the constant flow of $1.73 \mathrm{~mL} / \mathrm{minute}$

Oven temperature

$260^{\circ} \mathrm{C}$

$40^{\circ} \mathrm{C}$ (constant temperature for two minutes), with an increase of $8^{\circ} \mathrm{C} /$ minutes, to $150^{\circ} \mathrm{C}$ (constant temperature for two minutes), then $8^{\circ} \mathrm{C} /$ minutes to $250^{\circ} \mathrm{C}$ (constant temperature for two minutes), ending with a 20 minutes isothermal at $280^{\circ} \mathrm{C}$

GC running time 56 minutes

Mass spectra Taken at $70 \mathrm{eV}$, fragments from 10-1000 Da and a scan interval of 0.5 seconds 
TurboMass (Ver 5.2.0) Software was adopted to handle chromatograms and mass spectra. Database of NIST (National Institute Standard and Technology) was used to conduct interpretation on GCMS and relative peak area percentages of each component were calculated by comparing its average peak area to total areas.

\section{Results}

\section{Screening of gymnosperm solvent extracts}

Twelve gymnosperm solvent extracts recorded percent inhibition that was approximately $\geq 60 \%$ including 6 n-hexane extracts, 4 ethanol extracts and 2 methanol extracts (Table 1). Statistical analysis of solvent extract at 5 and $10 \%$ concentrations presented differences in percent inhibition values among treatments $(\mathrm{p}<0.05$, Table S1). Most of the plant solvent extracts significantly inhibited mycelial growth of Foc compared to the control treatments $(\mathrm{p}<0.05$, Table $S 2)$. The $P$. wallachiana methanol leaf extract $(66.67 \%)$ and $P$. wallachiana n-hexane cone extract $(66.66 \%)$ recorded highest inhibition against Foc. The n-hexane cone extracts from Cedrus deodara (66.07\%), P. smithiana $(66.07 \%)$, P. roxburghii $(62.97 \%)$, T. orientalis $(60.59 \%)$ also recorded higher inhibition against Foc. Moreover, methanol cone extract of $P$. smithiana $(65.47 \%)$, ethanol leaf $(62.38 \%)$ and cone $(65.83 \%)$ extracts from T. orientalis, $P$. wallachiana ethanol leaf extract $(60.12 \%)$ and $T$. orientalis $\mathrm{n}$-hexane leaf extract $(59.64 \%)$ also recorded higher percent inhibition values.

Table 1. Percent inhibition of Foc mycelia growth recorded for 5\% and 10\% of gymnosperm extracts prepared in n-hexane, ethanol and methanol solvents (Data presented as Mean $\pm S E$ )

\begin{tabular}{|c|c|c|c|c|c|c|}
\hline \multirow{2}{*}{ reatments } & \multicolumn{2}{|c|}{ n-Hexane (extracts) } & \multicolumn{2}{|c|}{ Ethanol (extracts) } & \multicolumn{2}{|c|}{ Methanol (extracts) } \\
\hline & $5 \%$ & $10 \%$ & $5 \%$ & $10 \%$ & $5 \%$ & $10 \%$ \\
\hline & & & & & & \\
\hline C. semp & $27.498 \pm 0.98^{\mathrm{def}}$ & $33.69 \pm 1.07^{\mathrm{ab}}$ & $23.45 \pm 1.10^{\text {ghi }}$ & $39.40 \pm 1.86^{\mathrm{WX}}$ & $33.81 \pm 0.74^{\mathrm{a}}$ & $49.64 \pm 1.44^{\mathrm{MN}}$ \\
\hline $\begin{array}{r}\text { C. semp } \\
\text { co }\end{array}$ & $32.26 \pm 2.14^{\mathrm{abc}}$ & $51.43 \pm 0.61^{\mathrm{KLM}}$ & $28.93 \pm 0.77^{\text {cde }}$ & $43.09 \pm 2.19^{\mathrm{STUV}}$ & $41.19 \pm 0.58^{\mathrm{UVWX}}$ & $54.17 \pm 0.90^{\mathrm{IJK}}$ \\
\hline T. orientalis leaf & $46.90 \pm 0.76^{\mathrm{NOPQR}}$ & $59.64 \pm 0.87^{\mathrm{DEFG}}$ & $47.74 \pm 0.35^{\mathrm{NOPQ}}$ & $62.38 \pm 1.27^{\mathrm{CD}}$ & $38.45 \pm 0.794^{\mathrm{XY}}$ & $46.08 \pm 2.14^{\mathrm{PQI}}$ \\
\hline T. orientalis cone & $55.95 \pm 1.67^{\mathrm{HIJ}}$ & $60.59 \pm 1.79^{\mathrm{DE}}$ & $50.24 \pm 0.97^{\mathrm{LMN}}$ & $65.83 \pm 0.35^{\mathrm{AB}}$ & $44.17 \pm 0.51^{\mathrm{RSTU}}$ & $56.07 \pm 1.18^{\mathrm{HIJ}}$ \\
\hline C. deodara leaf & $12.498 \pm 1.22^{\mathrm{m}}$ & $28.81 \pm 0.55^{\mathrm{de}}$ & $22.50 \pm 1.09^{\mathrm{hij}}$ & $38.21 \pm 0.58^{\mathrm{XYZ}}$ & $30.35 \pm 1.23^{\mathrm{bcd}}$ & $39.17 \pm 1.61^{\mathrm{wX}}$ \\
\hline C. deodara cone & $58.81 \pm 0.47^{\mathrm{EFGH}}$ & $66.07 \pm 1.54^{\mathrm{AB}}$ & $48.81 \pm 0.63^{\mathrm{MNOP}}$ & $60.47 \pm 0.55^{\mathrm{DE}}$ & $46.31 \pm 0.44^{\mathrm{OPQRS}}$ & $57.74 \pm 0.65^{\mathrm{EFC}}$ \\
\hline $\begin{array}{c}P . \text { wallachiana } \\
\text { leaf }\end{array}$ & $17.26 \pm 0.75^{1}$ & $25.83 \pm 4.58^{\mathrm{efgh}}$ & $49.17 \pm 0.44^{\mathrm{MNOP}}$ & $60.12 \pm 0.73^{\mathrm{DEF}}$ & $53.34 \pm 0.31^{\mathrm{JKL}}$ & $66.67 \pm 0.53^{\mathrm{A}}$ \\
\hline $\begin{array}{l}P . \text { wallachiana } \\
\text { cone }\end{array}$ & $56.31 \pm 1.17^{\mathrm{GHIJ}}$ & $66.66 \pm 1.25^{\mathrm{A}}$ & $18.81 \pm 1.49^{\mathrm{kl}}$ & $34.76 \pm 1.56^{\mathrm{a}}$ & $33.93 \pm 0.33^{\mathrm{a}}$ & $44.64 \pm 0.86^{\mathrm{QR}}$ \\
\hline P. smithiana leaf & $26.19 \pm 1.42^{\mathrm{efg}}$ & $34.40 \pm 0.87^{\mathrm{a}}$ & $20.95 \pm 0.98^{\mathrm{ijk}}$ & $35.24 \pm 1.10^{\mathrm{YZa}}$ & $24.28 \pm 2.192^{\text {fghi }}$ & $30.35 \pm 1.38^{\mathrm{bcd}}$ \\
\hline$P$. smithiana cone & $56.55 \pm 0.94^{\mathrm{GHIJ}}$ & $66.07 \pm 2.32^{\mathrm{AB}}$ & $42.02 \pm 0.97^{\mathrm{TUVW}}$ & $57.74 \pm 1.11^{\mathrm{EFGH}}$ & $51.78 \pm 0.42^{\mathrm{KLM}}$ & $65.47 \pm 0.37^{\mathrm{ABC}}$ \\
\hline P. roxburghii $\underset{\text { leaf }}{\text { rof }}$ & $42.26 \pm 0.997^{\mathrm{TUVW}}$ & $51.78 \pm 1.06^{\mathrm{KLM}}$ & $26.19 \pm 0.90^{\mathrm{efg}}$ & $34.88 \pm 0.81^{\mathrm{Za}}$ & $19.76 \pm 0.87^{\mathrm{jkl}}$ & $40.47 \pm 0.33^{\mathrm{VwX}}$ \\
\hline $\begin{array}{c}P \text {. roxburghii } \\
\text { cone }\end{array}$ & $52.14 \pm 1.80^{\mathrm{KLM}}$ & $62.97 \pm 0.93^{\mathrm{BCD}}$ & $1.55 \pm 1.12^{\mathrm{n}}$ & $17.02 \pm 1.36^{1}$ & $46.31 \pm 0.87^{\mathrm{OPQRS}}$ & $56.90 \pm 1.04^{\mathrm{FGHI}}$ \\
\hline
\end{tabular}

Mean values of treatments indicated by capital letter superscripts are significantly different from those of small letter superscripts and higher mean values are indicated by capital letter superscripts i.e. A, B,...Z while lower mean value are indicated by small letters i.e. $a, b, \ldots . z$. Values having same capital letters or small letters superscript do no differ statistically and the common letters (either capital or small letters) sharing between the treatments indicate non-significant difference $(\mathrm{LSD}=3.38)$ 


\section{The in vitro assay and pot experiment of selected solvent extracts}

a) In vitro assay

Selected solvent extracts recorded significant mycelial growth inhibition as compared to the positive control and solvent controls ( $\mathrm{p}<0.05$, Table S3). The fungicide control (propiconazole) and ethanol extact of $P$. wallachiana leaf recorded comparable mycelial inhibition. The P. wallachiana ethanol leaf extract $\left(\mathrm{Pw}_{\mathrm{EL}}=99.3 \%\right)$ followed by ethanol leaf extract of $T$. orientalis (ToEL $=85.9 \%$ ) were found best treatments against Foc mycelial growth using 50\% conc. $P$. wallachiana methanol leaf extract $\left(\mathrm{Pw}_{\mathrm{ML}}=82.8 \%\right)$, $P$. smithiana methanol cone extract $\left(\mathrm{Ps}_{\mathrm{MC}}=77.4 \%\right), P$. wallachiana $\mathrm{n}$-hexane cone extract $\left(\mathrm{Pw}_{\mathrm{HC}}=75.9 \%\right)$ and $T$. orientalis ethanol cone extract $\left(\mathrm{TO}_{\mathrm{EC}}=74.6 \%\right)$ also recorded significant inhibitory activity against Foc (Table 2). Only PwEL treatment was close to the inhibitory activity of propiconazole fungicide while rest of the treatments had lower inhibition values against Foc as compared to the fungicide treatment.

Table 2. Percent inhibition of Foc mycelia growth using $50 \%$ concentrations of the selected solvent extracts and growth parameter values and severity scoring recorded at the end of greenhouse assay (Data presented as Mean $\pm S E$ )

\begin{tabular}{|c|c|c|c|c|c|}
\hline Treatment & $\begin{array}{c}\text { Percent } \\
\text { Inhibition } \\
(\mathrm{LSD}=4.28)\end{array}$ & $\begin{array}{c}\text { Length of } \\
\text { Pseudostem in } \mathbf{~ c m ~} \\
(\mathrm{LSD}=3.6)\end{array}$ & $\begin{array}{c}\begin{array}{c}\text { Leaf length in } \\
\text { cm }\end{array} \\
(\mathrm{LSD}=4.4)\end{array}$ & $\begin{array}{c}\text { Leaf width } \\
\text { in cm (LSD } \\
=1.9)\end{array}$ & $\begin{array}{c}\text { Severity } \\
\text { scores } \\
(\mathrm{LSD}=0.5)\end{array}$ \\
\hline Positive control & $0^{\mathrm{I}}$ & $27.69 \pm 1.2^{\mathrm{I}}$ & $23.83 \pm 1.4^{\mathrm{F}}$ & $9.91 \pm 0.3^{\mathrm{F}}$ & $5 \pm 0.0^{\mathrm{A}}$ \\
\hline Hexane control & $0^{\mathrm{I}}$ & $27.18 \pm 1.1^{\mathrm{I}}$ & $26.42 \pm 1.1^{\mathrm{EF}}$ & $12.45 \pm 0.5^{\mathrm{E}}$ & $5 \pm 0.0^{\mathrm{A}}$ \\
\hline Ethanol control & $0^{\mathrm{I}}$ & $29.21 \pm 0.7^{\mathrm{HI}}$ & $27.69 \pm 0.39^{\mathrm{DEF}}$ & $13.12 \pm 0.1^{\mathrm{DE}}$ & $5 \pm 0.0^{\mathrm{A}}$ \\
\hline Methanol control & $0^{\mathrm{I}}$ & $29.72 \pm 0.9^{\mathrm{GHI}}$ & $27.69 \pm 0.9^{\mathrm{DEF}}$ & $12.78 \pm 0.4^{\mathrm{DE}}$ & $5 \pm 0.0^{\mathrm{A}}$ \\
\hline Fungicide control & $100 \pm 0.0^{\mathrm{A}}$ & $33.02 \pm 0.7^{\mathrm{EFG}}$ & $35.14 \pm 1.8^{\mathrm{AB}}$ & $16.00 \pm 1.0^{\mathrm{BC}}$ & $3 \pm 0.0^{\mathrm{EF}}$ \\
\hline $\begin{array}{l}\text { Hexane cone extract of } P \text {. } \\
\text { wallachiana }\left(\mathrm{PwHC}_{\mathrm{HC}}\right)\end{array}$ & $75.9 \pm 1.4^{\mathrm{C}}$ & $37.42 \pm 0.6^{\mathrm{BCD}}$ & $31.33 \pm 0.4^{\mathrm{BCD}}$ & $14.65 \pm 0.4^{\mathrm{CD}}$ & $3 \pm 0.0^{\mathrm{EF}}$ \\
\hline $\begin{array}{l}\text { Methanol leaf extract of } P \text {. } \\
\text { wallachiana (PwML) }\end{array}$ & $82.8 \pm 1.2^{\mathrm{B}}$ & $39.79 \pm 0.4^{\mathrm{ABC}}$ & $34.04 \pm 1.2^{\mathrm{ABC}}$ & $16.17 \pm 0.4^{\mathrm{BC}}$ & $3 \pm 0.0^{\mathrm{EF}}$ \\
\hline $\begin{array}{c}\text { Hexane cone extract of } P \text {. } \\
\text { smithiana (PSHC) }\end{array}$ & $55.9 \pm 1.2^{\mathrm{H}}$ & $38.9 \pm 1.1^{\mathrm{ABCD}}$ & $33.44 \pm 1.8^{\mathrm{BC}}$ & $16.26 \pm 0.8^{\mathrm{ABC}}$ & $3.67 \pm 0.3^{\mathrm{CD}}$ \\
\hline $\begin{array}{l}\text { Hexane cone extract of } C \text {. Deodara } \\
\text { (CdHC) }\end{array}$ & $66.7 \pm 0.7^{\mathrm{EF}}$ & $39.37 \pm 0.0^{\mathrm{ABC}}$ & $33.44 \pm 1.1^{\mathrm{BC}}$ & $15.66 \pm 0.5^{\mathrm{BC}}$ & $3 \pm 0.0^{\mathrm{EF}}$ \\
\hline $\begin{array}{c}\text { Ethanol cone extract of } T \text {. } \\
\text { orientalis (ToEc) }\end{array}$ & $74.6 \pm 1.9^{\mathrm{CD}}$ & $38.35 \pm 1.0^{\mathrm{ABCD}}$ & $32.60 \pm 1.1^{\mathrm{BC}}$ & $16.34 \pm 0.7^{\mathrm{ABC}}$ & $3 \pm 0.0^{\mathrm{EF}}$ \\
\hline $\begin{array}{l}\text { Methanol cone extract of } P \text {. } \\
\text { Smithiana (PSMC) }\end{array}$ & $77.4 \pm 0.9^{\mathrm{C}}$ & $31.37 \pm 3.9^{\mathrm{GH}}$ & $29.63 \pm 3.7^{\mathrm{CDE}}$ & $14.73 \pm 1.6^{\mathrm{CD}}$ & $3 \pm 0.0^{\mathrm{EF}}$ \\
\hline $\begin{array}{c}\text { Hexane cone extract of } P \text {. } \\
\text { roxburghi }\left(\operatorname{Pr}_{\mathrm{HC}}\right)\end{array}$ & $70.8 \pm 0.8^{\mathrm{DE}}$ & $38.10 \pm 0.0^{\mathrm{ABCD}}$ & $34.63 \pm 1.4^{\mathrm{AB}}$ & $17.36 \pm 0.5^{\mathrm{AB}}$ & $3.67 \pm 0.3^{\mathrm{CD}}$ \\
\hline $\begin{array}{c}\text { Ethanol leaf extract of } T \text {. orientalis } \\
\text { (ToEL) }\end{array}$ & $85.9 \pm 0.8^{\mathrm{B}}$ & $40.22 \pm 0.8^{\mathrm{AB}}$ & $33.27 \pm 0.7^{\mathrm{BC}}$ & $16.00 \pm 0.4^{\mathrm{BC}}$ & $2.67 \pm 0.3^{\mathrm{FG}}$ \\
\hline $\begin{array}{c}\text { Hexane cone extract of } T \text {. orientalis } \\
\text { (Тонс) }\end{array}$ & $63.2 \pm 0.9^{\mathrm{FG}}$ & $31.75 \pm 0.7 \mathrm{~F}^{\mathrm{GH}}$ & $31.33 \pm 2.2^{\mathrm{BCD}}$ & $15.15 \pm 0.4^{\mathrm{C}}$ & $4 \pm 0.0^{\mathrm{BC}}$ \\
\hline $\begin{array}{c}\text { Ethanol cone extract of } C \text {. Deodara } \\
\left(\mathrm{Cd}_{\mathrm{EC}}\right)\end{array}$ & $62.9 \pm 1.9^{\mathrm{FG}}$ & $35.31 \pm 0.9^{\mathrm{DEF}}$ & $31.50 \pm 1.7^{\mathrm{BCD}}$ & $15.15 \pm 0.7^{\mathrm{C}}$ & $3.33 \pm 0.3^{\mathrm{DE}}$ \\
\hline $\begin{array}{c}\text { Ethanol leaf extract of } \boldsymbol{P} . \\
\text { wallachiana (PwEL) }\end{array}$ & $99.3 \pm 0.5^{\mathrm{A}}$ & $41.66 \pm 1.2^{\mathrm{A}}$ & $38.27 \pm 0.7^{\mathrm{A}}$ & $18.20 \pm 0.8^{\mathrm{A}}$ & $2.33 \pm 0.3^{\mathrm{G}}$ \\
\hline $\begin{array}{c}\text { Hexane leaf extract of } T \text {. orientalis } \\
\text { (ToHL) }\end{array}$ & $59.9 \pm 4.8^{\mathrm{GH}}$ & $36.41 \pm 1.4^{\mathrm{CDE}}$ & $30.06 \pm 1.1^{\mathrm{CDE}}$ & $15.15 \pm 0.7^{\mathrm{C}}$ & $4.33 \pm 0.3^{\mathrm{B}}$ \\
\hline
\end{tabular}

Values with same superscript letters within an individual column do no differ statistically. Common letter sharing indicate non-significant difference between the treatments 


\section{b) Pot experiment}

Banana plants in control treatments recorded visual symptoms of disease earlier compared to the majority of the treatments of solvent extract. Except fungicide control, disease severity index (DSI) of the other controls were higher compared to extract treatments. At the end of experiment, all the controls excluding fungicide control had $100 \%$ DSI and highest (5) severity scores. Minimum DSI was recorded for ethanol leaf extract of $P$. wallachiana ( $\mathrm{Pw} \mathrm{wL}_{\mathrm{EL}}$ ) i.e. $46.6 \%$, followed by ethanol leaf extract of T. orientalis (Toel) i.e. 53.3\% DSI (Fig. 1). Statistical analysis presented differences in the values of severity scores ( $\mathrm{p}<0.05$, Table S4), pseudostem length ( $\mathrm{p}<0.05$, Table S5), leaf width ( $\mathrm{p}<0.05$, Table S6) and leaf length ( $\mathrm{p}<0.05$, Table S7) among treatments. Lowest severity scoring (2.33) and highest pseudostem length $(41.66 \mathrm{~cm})$, leaf width $(18.2 \mathrm{~cm})$ and leaf length $(38.27 \mathrm{~cm})$ had also been recorded for PwEL (Table 2).
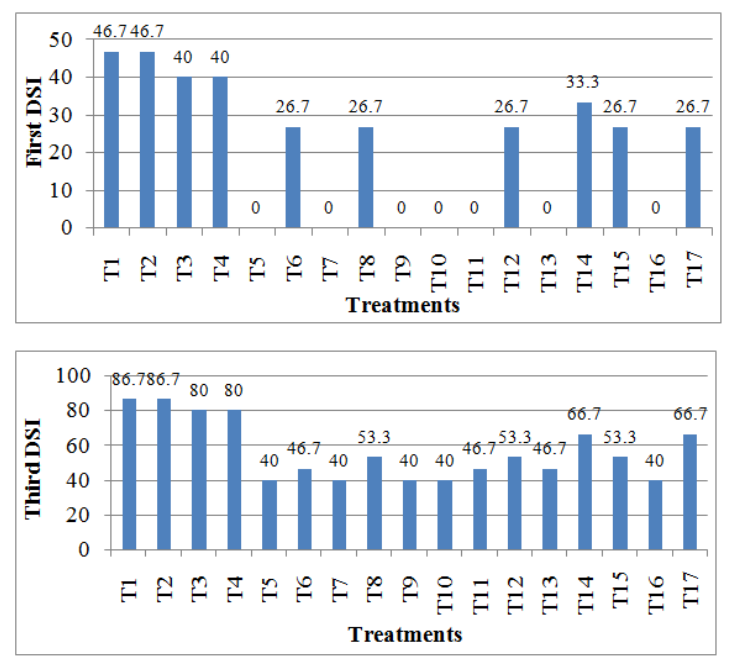
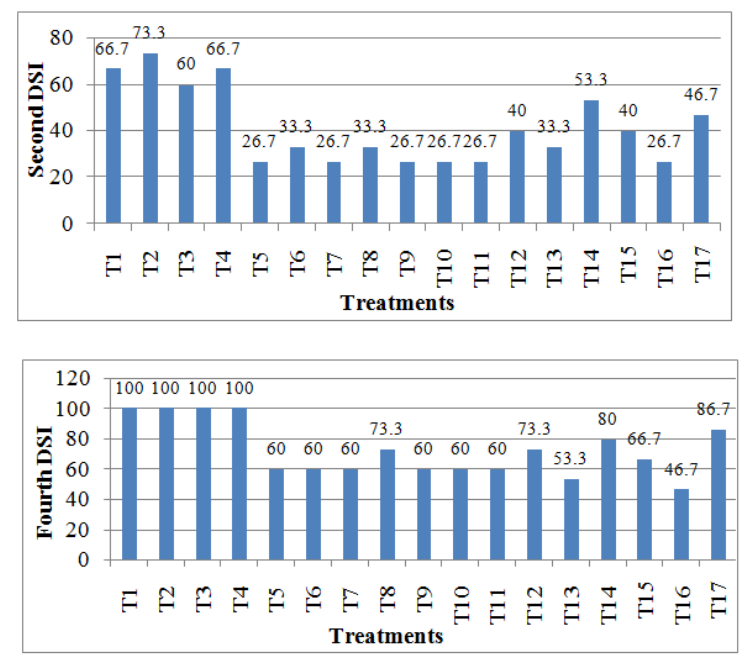

Figure 1. Progression in the Disease severity index (DSI) of different root dipping treatments, including positive control $\left(T_{1}\right)$; solvent controls $\left(T_{2}-T_{4}\right)$; negative control $\left(T_{5}\right)$ and solvent extract treatments $\left(T_{6}-T_{17}\right)$, recorded during pot experiment. ( $T_{1}$ : Positive control, $T_{2}: n$-Hexane control, $T_{3}$ : Ethanol control, $T_{4}$ : Methanol control, $T_{5}$ : Negative (Fungicide) control, $T_{6:} n$ Hexane cone extract of $P$. wallachiana, $T_{7}:$ Methanol leaf extract of $P$. wallachiana, $T_{8}: n$ Hexane cone extract of $P$. smithiana, $T_{9:}$-Hexane cone extract of $C$. deodara, $T_{10}$ : Ethanol cone extract of $T$. orientalis, $T_{11}$ : Methanol cone extract of $P$. smithiana, $T_{12}: n$-Hexane cone extract of $P$. roxburghi, $T_{13}$ : Ethanol leaf extract of $T$. orientalis, $T_{14}$ : $n$-Hexane cone extract of

T. orientalis, $T_{15}$ : Ethanol cone extract of $C$. deodara, $T_{16}$ : Ethanol leaf extract of $P$. wallachiana, $T_{17}:$ n-Hexane leaf extract of $T$. orientalis)

\section{GC-MS analysis for $P_{E L}$ and ToEL}

The total ion chromatogram (TIC) of PwEL detected seven compounds having retention time ranging between 19.1 to 41.6 minutes (Fig. 2). The delta-Cadinene, Vitamin C, cis-9-Hexadecenal, Oleic acid, 1-Naphthalenecarboxylic acid, 1- Naphthalenepentanoic acid and Hexatriacontyl trifluoroacetate were present in PwEL. The 1-Naphthalenecarboxylic acid, Vitamin $\mathrm{C}$ and Hexatriacontyl trifluoroacetate were detected with high peak area percent (Table 3). Total ion chromatogram of ToEL also detected seven compounds having retention time ranging between 21.06 to 36.2 minutes (Fig. 2). The Cedrol, cis-9-Hexadecenal, Podocarp-7-en, Pimaric acid, 
1-Phenanthrenecarboxylic acid, 1-Naphthalenepentanoic acid and 1-Naphthalenecarboxylic acid were detected in ToEL. The 1-Naphthalenecarboxylic acid (Hardwickiic acid), Cedrol and 1-Naphthalenepentanoic acid were found with high percentage of peak area (Table 3). The 1-Naphthalenepentanoic acid, 1-Naphthalenecarboxylic acid and cis-9-Hexadecenal were noticed in the two extracts (Fig. 3).
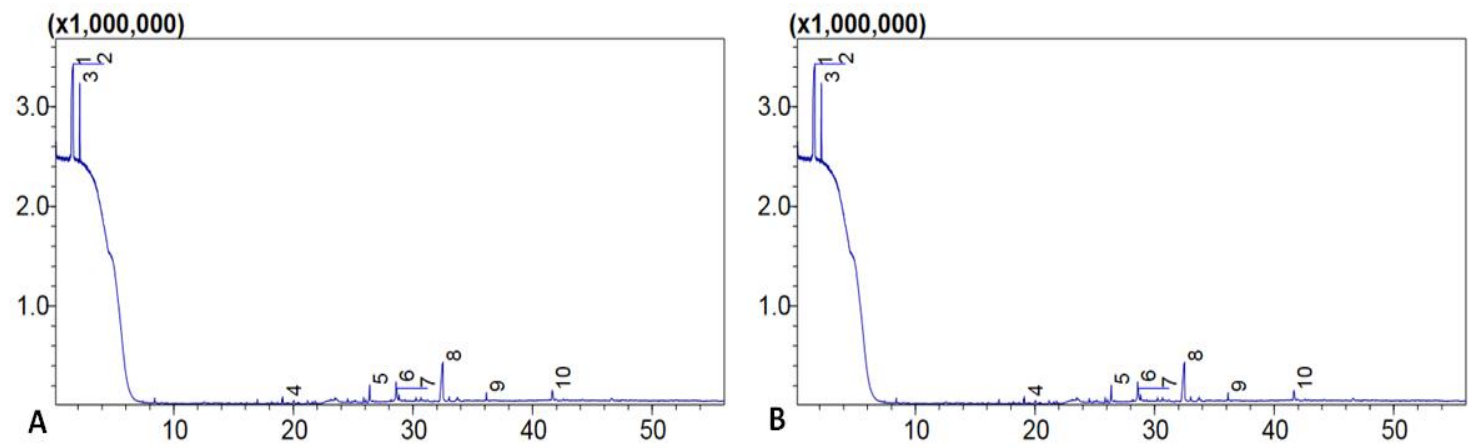

Figure 2. Total ion chromatogram of $P w_{E L}(A)$ and $T_{E L}(B)$ obtained through GCMS analysis

Table 3. Compounds detected in $P w_{E L}$ and To $o_{E L}$ with GC-MS analysis

\begin{tabular}{|c|c|c|c|c|c|c|c|c|}
\hline \multirow[b]{2}{*}{ Peak } & \multicolumn{4}{|c|}{ Compounds in PweL } & \multicolumn{4}{|c|}{ Compounds in ToEL } \\
\hline & $\mathbf{R}_{\mathbf{T}}$ & Area \% & $\begin{array}{l}\text { Name/ Formula/ } \\
\text { Molecular weight }\end{array}$ & Chemical group & $\mathbf{R}_{\mathbf{T}}$ & Area \% & $\begin{array}{l}\text { Name/ Formula/ } \\
\text { Molecular weight }\end{array}$ & $\begin{array}{l}\text { Chemical } \\
\text { group }\end{array}$ \\
\hline 1 & 1.55 & 41.8 & Ethanol/ $\mathrm{C}_{2} \mathrm{H}_{6} \mathrm{O} / 46$ & Solvent & 1.59 & 55.0 & Ethanol/ $\mathrm{C}_{2} \mathrm{H}_{6} \mathrm{O} / 46$ & Solvent \\
\hline 2 & 1.59 & 11.4 & Ethanol/ $\mathrm{C}_{2} \mathrm{H}_{6} \mathrm{O} / 46$ & Solvent & 2.14 & 11.0 & $\begin{array}{c}\text { Chloroform/ } \mathrm{CHCl}_{3} / \\
118\end{array}$ & Solvent \\
\hline 3 & 2.14 & 10.3 & $\begin{array}{l}\text { Chloroform/ } \mathrm{CHCl}_{3} / \\
118\end{array}$ & Solvent & 21.06 & 3.9 & Cedrol/ $\mathrm{C}_{15} \mathrm{H}_{26} \mathrm{O} / 222$ & $\begin{array}{l}\text { Sesquiterpene } \\
\text { alcohol }\end{array}$ \\
\hline 4 & 19.11 & 1.0 & $\begin{array}{l}\text { delta-Cadinene/ } \mathrm{C}_{15} \mathrm{H}_{24} / \\
204\end{array}$ & $\begin{array}{l}\text { Cadinene family } \\
\text { of sesquiterpines }\end{array}$ & 28.60 & 2.9 & $\begin{array}{l}\text { cis-9-Hexadecenal/ } \\
\mathrm{C}_{16} \mathrm{H}_{30} \mathrm{O} / 238\end{array}$ & $\begin{array}{l}\text { Palmitole } \\
\text { aldehyde }\end{array}$ \\
\hline 5 & 26.39 & 3.1 & $\begin{array}{c}\text { Vitamin } \mathrm{C} / \mathrm{C}_{38} \mathrm{H}_{68} \mathrm{O}_{8} / \\
652\end{array}$ & Ester & 28.66 & 1.1 & $\begin{array}{c}\text { Podocarp-7-en-3-one, } \\
\text { 13.beta.-methyl-1/ } \\
\mathrm{C}_{20} \mathrm{H}_{30} \mathrm{O} / 286\end{array}$ & $\begin{array}{l}\text { Diterpenoid/ } \\
\text { meroterpene } \\
\text { natural phenol }\end{array}$ \\
\hline 6 & 28.59 & 3.7 & $\begin{array}{l}\text { cis-9-Hexadecenal/ } \\
\mathrm{C}_{16} \mathrm{H}_{30} \mathrm{O} / 238\end{array}$ & $\begin{array}{l}\text { Palmitole } \\
\text { Aldehyde }\end{array}$ & 30.61 & 1.7 & $\begin{array}{l}\text { Podocarp-7-en-3-one, } \\
\text { 13.beta.-methyl-1/ } \\
\mathrm{C}_{20} \mathrm{H}_{30} \mathrm{O} / 286\end{array}$ & $\begin{array}{l}\text { Diterpenoid/ } \\
\text { meroterpene } \\
\text { natural phenol }\end{array}$ \\
\hline 7 & 28.64 & 1.4 & $\begin{array}{c}\text { Oleic acid/ } \mathrm{C}_{18} \mathrm{H}_{34} \mathrm{O}_{2} / \\
282\end{array}$ & $\begin{array}{c}\text { Monounsaturated } \\
\text { (omega-9) fatty } \\
\text { acid }\end{array}$ & 31.26 & 2.3 & $\begin{array}{c}\text { Pimaric acid/ } \mathrm{C}_{20} \mathrm{H}_{30} \mathrm{O}_{2} / \\
302\end{array}$ & $\begin{array}{l}\text { Carboxylic } \\
\text { acid from the } \\
\text { resin acid } \\
\text { group }\end{array}$ \\
\hline 8 & 2.51 & 22.6 & $\begin{array}{c}1- \\
\text { Naphthalenecarboxylic } \\
\text { acid / C20H28O3/ } 316\end{array}$ & $\begin{array}{l}\text { Clerodane } \\
\text { diterpenoid }\end{array}$ & 32.00 & 3.3 & $\begin{array}{c}1- \\
\text { Phenanthrenecarboxylic } \\
\text { acid / } \mathrm{C}_{2} \mathrm{H}_{32} \mathrm{O}_{2} / 316\end{array}$ & $\begin{array}{l}\text { Methyl ester/ } \\
\text { Neoabietic acid }\end{array}$ \\
\hline 9 & 36.14 & 1.5 & $\begin{array}{c}\text { 1-Naphthalenepentanoic } \\
\text { acid/ } \mathrm{C}_{22} \mathrm{H}_{36} \mathrm{O}_{4} / 364\end{array}$ & Methyl ester & 32.47 & 8.9 & $\begin{array}{c}1- \\
\text { Naphthalenecarboxylic } \\
\text { acid } / \mathrm{C}_{20} \mathrm{H}_{28} \mathrm{O}_{3} / 316\end{array}$ & $\begin{array}{l}\text { Clerodane } \\
\text { diterpenoid }\end{array}$ \\
\hline 10 & 41.64 & 3.1 & $\begin{array}{l}\text { Hexatriacontyl } \\
\text { trifluoroacetate/ } \\
\mathrm{C}_{38} \mathrm{H}_{73} \mathrm{~F}_{3} \mathrm{O}_{2} / 618\end{array}$ & Alcohol acetate & 36.21 & 9.8 & $\begin{array}{c}\text { 1-Naphthalenepentanoic } \\
\text { acid/ } \mathrm{C}_{22} \mathrm{H}_{36} \mathrm{O}_{4} / 364\end{array}$ & Methyl ester \\
\hline
\end{tabular}




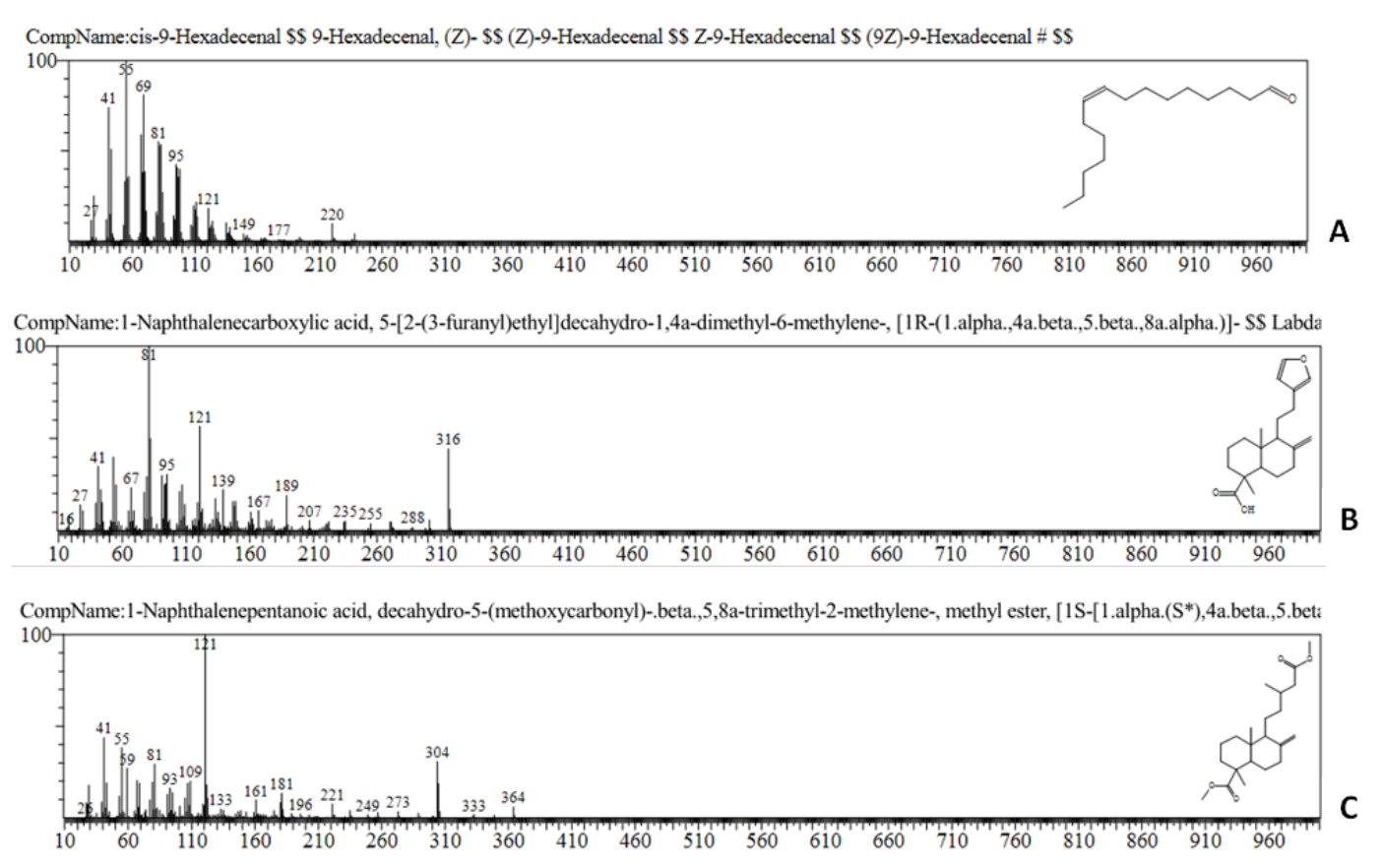

Figure 3. Standard molecular spectra of cis-9-Hexadecenal (A), 1-Naphthalenecarboxylic acid $(B)$ and 1-Naphthalenepentanoic acid $(C)$ detected in $P w_{E L}$ and To $o_{E L}$

\section{Discussion}

Modern science is using huge array of plants because of their antimicrobial traits, which are attributed to compounds synthesized in the secondary metabolism of the plant (Nascimento, 2000). Terpenoids, tannins, ligans, steroids, alkaloids, phenols, glycosides, and sugar derivatives are noted as secondary metabolites in the gymnosperms (Harborne and Baxter, 2001). Plant secondary metabolites structure has been optimized during evolutionary process so that they can interfere with microbial molecular targets thus acting as a defense mechanism (Wink et al., 2012). Plant extracts disrupts the normal cell functioning of microbes, thus affecting important steps in the pathogenic process (Gupta and Birdi, 2017). Botanicals comprises such phytocompounds that cause morphological alterations in fungal hyphae which results to shriveling of hyphae, protoplast leakage and vacuolations coagulation (Soylu et al., 2006). The modes of action of phytoproducts on cells of fungi are thought to be membrane rupture in cytoplasm, granulation of cytoplasm, inhibition of extracellular and intracellular synthesis of enzymes (Cowan, 1999). Result of preliminary in vitro screening for gymnosperm solvent extracts recorded significant inhibition of Foc compared to the control treatments that clearly indicates antifungal efficacy of the tested gymnosperm plants. Joshi and Sati (2012) described antimicrobial efficacy of botanicals from many plant species belonging to various families of gymnosperms and current study is in accordance with their findings. Although, antimicrobial potential of gymnosperm botanicals has been reported against various tested pathogens but their antifungal efficacy against Foc is never been tested. This study first time reports the efficacy of solvent extracts of selected gymnosprem plants against Foc. It was noted that some extracts had similar inhibition activity with all three solvent types while some extracts recorded different inhibition potential using three solvents. This might be because of the variable content of phytochemicals in each plant extract and 
because of different extraction potentials of each solvent. Khoddami et al. (2013) described that type of plant/plant part and extraction solvent are reason for the variation of phytochemical formation in various extracts. Altemimi et al. (2017) noticed solvent effects on the phytochemical constituent profiles and antioxidant activities of different plant extracts.

Twelve solvent extracts selected through antifungal screening and further evaluated in the in vitro assay and green house assay, reported efficacy of $\mathrm{Pw}_{\mathrm{EL}}$ comparable to fungicide (propiconazole) treatment. Highest mycelial inhibition against Foc indicated that PWEL was the most efficient solvent extract, suggesting presence of comparatively higher amount of effective phytochemicals in their composition, which effectively restricted and inhibited Foc mycelial growth. Sharma et al. (2018) recorded insecticidal and antimicrobial activities of $P$. wallachiana leaf extracts. Sharma et al. (2015) also noted antioxidant and antibacterial activities of alcoholic extracts of $P$. wallachiana leaf. Highest values in growth parameters and minimum DSI were recorded for PwEL followed by ToEL compared to the control treatments in green house assay. Huang et al. (2012) and Gopi and Thangavelu (2014) noted that botanical treatments effectively suppressed Fusarium wilt in green house experiments.

GCMS analysis of PwEL and ToEL detected seven compounds in each extract. Some reported compounds have well-known antimicrobial efficacies i.e. Oleic acid, 1-Naphthalenepentanoic acid, cis-9-Hexadecenal, delta-Cadinene, 1-Naphthalenecarboxylic acid (Hardwickiic acid), Cedrol and 1-Phenanthrene carboxylic acid. Dar et al. (2012) reported delta-Cadiene as one of the chemical constituent detected from the essential oil of $P$. wallachiana needle. Joshi et al. (2016) described thirty-eight compounds in the $P$. wallachiana methanolic leaf extracts including Vitamin C. Anburaj et al. (2016) reported Vitamin $\mathrm{C}$ as antioxidant, immunomodulator and anticancer. Jang et al. (2016) noted antimicrobial activity from Eleutherococcus senticosus essential oil containing delta-Cadinene as one of its compositional compounds. The cis-9-Hexadecenal has been reported for its antimicrobial activity (Mujeeb et al., 2014). Awa et al. (2012) noticed antibacterial activity of Oleic acid. McChesney et al. (1991) described antimicrobial potential of 1-Naphthalenecarboxylic acid (Hardwickiic acid) isolated from the Croton sonderianus. Similarly, Kuete et al. (2007) also revealed antimicrobial potential of compounds (including Hardwickiic acid) extracted from Irvingia gabonensis stem bark.

Khubeiz et al. (2016) and Moawad and Amin (2019) noted sesquiterpenoids and monoterpenes as chemical components from leaf essential oil of $T$. orientalis containing Cedrol as one of the major component recording significant antibacterial activity. Madhumitha et al. (2012) described insecticidal efficacy of aqueous extract fruit peel of Annona squamosa against parasites (blood feeding). Its constitutional chemical compounds included Podocarp-7-en. Naikwadi et al. (2017) revealed antibacterial potential of Vetiveria zizanioides root extracts containing 1-Phenanthrene carboxylic acid, Phenanthrene carboxylic acid and Naphthalene pentanoic acid as major bioactive constituents. Manimegalai et al. (2011) isolated compounds from T. orientalis bark that were antibacterial and separation by TLC and GCMS disclosed presence of Phenanthrene carboxylic acid in it. Ali et al. (2011) reported anti-inflammatory and antibacterial potency of Pimaric acid.

Compounds that were detected through GCMS in PwEL and ToEL might be the probable phytoconstituents for Foc control in the in vitro and pot assays. These phytochemicals either through stimulating host's defence response by activating PR-proteins or directly 
toxifying Foc, inhibited mycelial amelioration in the pot and in vitro experiments respectively. Zhang et al. (2013) described that volatiles derived from plant source displayed higher inhibition potential on Foc and the compound i.e. 2-methyl-2 pentenal, completely inhibited mycelial growth of Foc. Gopi and Thangavelu (2014) reported lipid compound in botanical treatment as effective constituent that significantly suppressed Panama disease under greenhouse assay. Guimaraes et al. (2011) and Siripornvisal (2010) also noted antifungal potential of volatile compounds against Foc inhibiting spore germination and mycelial growth. Three compounds i.e. 1-Naphthalenepentanoic acid, 1-Naphthalenecarboxylic acid and cis-9-Hexadecenal, were revealed in both extracts that might be the potential active components of PwEL and ToEL. According to our knowledge, cis-9-Hexadecenal, 1-Naphthalenecarboxylic acid (Hardwickiic acid), Oleic acid, Hexatriacontyl trifluoroacetate and 1-Naphthalenepentanoic acid are noticed in PwEL, for the first time, through current study. Moreover, Podocarp-7-en, cis-9-Hexadecenal, 1-Phenanthrenecarboxylic acid, Pimaric acid, 1-Naphthalenepentanoic acid and 1-Naphthalenecarboxylic acid are also reported first time in Toel as most of the phytochemical studies on Thuja orientalis are focused on its essential oil which only reports cedrol presence.

\section{Conclusion}

A baseline study that for the first time addresses Fusarium wilt dilemma using botanicals and reports antifungal efficacy of selected gymnosperms against Foc. Even though variability was found in the inhibitory activity of different gymnosperm solvent extracts, However, few solvent extracts recorded good efficacy against Foc both in the in vitro and pot experiments. The ethanolic leaf extract of $P$. wallachiana $\left(\mathrm{Pw}_{\mathrm{EL}}\right)$ and the ethanolic leaf extract of $T$. orientalis (Toes) were evidenced through GCMS to have bountiful sources of such valuable phytochemicals that can replace conventional chemicals and fungicides that are used for the management of Panama wilt disease. It is therefore, strongly recommended that the efficient gymnosperm extract should be further explored through spectrophotometric and chromatographic analysis. Moreover, compound detected through GCMS should be individually evaluated against Foc using in vitro and green house experiments.

Acknowledgements. Author is thankful to Higher Education Commission, for their financial support. Special thanks to Plant and Environmental Protection department, NARC and National Center of Physics (NCP), Quaid i Azam University (QAU), Islamabad for arrangement of laboratory facilities for current study.

\section{REFERENCES}

[1] Ali, F., Sangwan, P. L., Koul, S., Pandey, A., Bani, S., Abdullah, S. T., Sharma, P. R., Kitchlu, S., Khan, I. A. (2011): 4-epi-Pimaric acid: a phytomolecule as a potent antibacterial and anti-biofilm agent for oral cavity pathogens. - Eur J Clin Microbiol Infect Dis. doi: 10.1007/s10096-011-1287-x.

[2] Altemimi, A., Lakhssassi, N., Baharlouei, A., Watson, D. G., Lightfoot, D. A. (2017): Phytochemicals: Extraction, isolation and identification of bioactive compounds from plant extracts. - Plants 6(42). doi:10.3390/plants6040042. 
[3] Anburaj, G., Marimuthu, M., Rajasudha, V., Manikandan, D. R. (2016): Phytochemical screening and GC-MS analysis of ethanolic extract of Tecoma stans (Family: Bignoniaceae) Yellow bell flowers. - Journal of Pharmacognosy and Phytochemistry 5(4): 172-175.

[4] Awa, E. P., Ibrahim, S., Ameh, D. A. (2012): GC/MS analysis and antimicrobial activity of diethyl ether fraction of methanolic extract from the stem bark of Annona senegalensis Pers. - Int J Pharm Sci Res 3: 4213-4218.

[5] Bajpai, V. K., Kang, S. C. (2010): Antifungal activity of leaf essential oil and extracts of Metasequoia glyptostroboides Miki ex Hu. - J Am Oil Chem Soc 87: 327-336. doi: 10.1007/s11746-009-1500-6.

[6] Cowan, M. M. (1999): Plants products as antimicrobial agents. - Clinical Microbiology Reviews 12: 564-582.

[7] Dar, M. Y., Shah, W. A., Mubashir, S., Rather, M. A. (2012): Chromatographic analysis, anti-proliferative and radical scavenging activity of Pinus wallichiana essential oil growing in high altitude areas of Kashmir, India. - Phytomedicine 19(13): 1228-1233. https:// doi.org/10.1016/j.phymed.2012.07.015.

[8] Dita, M., Barquero, M., Heck, D., Mizubuti, E. S. G., Staver, C. P. (2018): Fusarium Wilt of Banana: Current knowledge on epidemiology and research needs toward sustainable disease management. - Front Plant Sci 9: 1468. doi: 10.3389/ fpls. 2018.01468.

[9] Doughari, J. H., Human, I. S., Bennade, S., Ndakidemi, P. A. (2009): Phytochemicals as chemotherapeutic agents and antioxidants: Possible solution to the control of antibiotic resistant verocytotoxin producing bacteria. - Journal of Medicinal Plants Research 3(11): 839-848.

[10] Drenth, A., Guest, D. I. (2016): Fungal and Oomycete diseases of tropical tree fruit crops. - Annu Rev Phytopathol 54: 373-395. doi: 10.1146/annurev-phyto-080615-095944.

[11] Ghag, S. B., Shekhawat, U. K. S., Ganapathi, T. R. (2015): Fusarium wilt of banana: Biology, epidemiology and management. - International Journal of Pest Management 61(3): 250-263. doi: 10. 1080/ 09670874. 2015.1043972.

[12] Gnanasekaran, P., Salique, S. M., Panneerselvam, A., Umamagheswari, K. (2015): In vitro Biological control of Fusarium oxysporum f. sp. cubense by using some Indian medicinal plants. - Int J Curr Res Aca Rev 3(11): 107-116.

[13] Gopi, M., Thangavelu, R. (2014): Suppression of Fusarium wilt disease of banana by Zimmu (Allium cepa L. x Allium sativum L.) leaf extract. - African Journal of Microbiology Reasearch 8(31): 2904-2915. doi: 10.5897/AJMR2014.6810.

[14] Guimaraes, L. G. L., Cardoso, M. G., Souza, P. E., Andrade, J., Vieira, S. S. (2011): Atividades antioxidante e fungitóxica do óleo essencial de capim-limão e do citral. Ciência agronômica 42(2): 464-472. http://dx.doi.org/10. 1590/S18066902011000200028.

[15] Gupta, P. D., Birdi, T. J. (2017): Development of botanicals to combat antibiotic resistance. - Journal of Ayurveda and Integrative Medicine 8: 266-275.

[16] Harborne, J. B., Baxter, H. (2001): The chemical dictionary of economic plants. - Wiley and Sons, Chichester, 582p.

[17] Huang, Y. H., Wang, R. C., Li, C. H., Zuo, C. W., Wei, Y. R., Zhang, L., Yi, G. J. (2012): Control of Fusarium wilt in banana with Chinese leek. - NIH (PMC) 134(1): 87-95. doi:10.1007/s10658-012-0024-3.

[18] Jang, D., Lee, J., Eom, S. H., Lee, S. M., Gil, J., Lim, H. B., Hyun, T. K. (2016): Composition, antioxidant and antimicrobial activities of Eleutherococcus senticosus fruit extracts. - Journal of Applied Pharmaceutical Science 6(03): 125-130. doi: 10.7324/JAPS.2016.60322.

[19] Joshi, S., Sati, S. C. (2012): Antifungal Potential of Gymnosperms: A Review. - In: Sati, S. C., Belwal, M. (eds.) Contribution to the mycological progress. Daya publishing house, pp. 333-345. doi: 10. 13140/ RG.2.1. 3408.0084. 
[20] Joshi, S., Rai, N., Kumar, N. (2016): Gas Chromatography-Mass Spectroscopy analysis of phytochemical constituents of methanolic extract of needles of Pinus wallachiana. - Asian J Pharm Clin Res 9(2): 55-57.

[21] Karpagasundari, C., Kulothungan, S. (2014): Analysis of bioactive compounds in Physalis minima leaves using GC MS, HPLC, UV-VIS and FTIR techniques. - Journal of Pharmacognosy and Phytochemistry 3(4): 196-201.

[22] Khoddami, A., Wilkes, M. A., Roberts, T. H. (2013): Techniques for analysis of plant phenolic compounds. - Molecules 18: 2328-2375. doi: 10.3390/ molecules 18022328.

[23] Khubeiz, M. J., Mansour, G., Zahraa, B. (2016): Antibacterial and phytochemical investigation of Thuja orientalis (1.) leaves essential oil from Syria. - International Journal of Current Pharmaceutical Review and Research 7(5): 243-247.

[24] Kuete, V., Wabo, G. F., Ngameni, B., Mbaveng, A. T., Metuno, R., Etoa, F-X., Ngadjui, B. T., Beng, V. P., Meyer, J. J. M., Lall, N. (2007): Antimicrobial activity of the methanolic extract, fractions and compounds from the stem bark of Irvingia gabonensis (Ixonanthaceae). - Journal of Ethnopharmacology 114(1): 54-60. https:// doi.org/ 10.1016 /j.jep.2007.07.025.

[25] Mackesy, D., Sullivan, M. (2015): CPHST Pest Datasheet for Fusarium oxysporum f. sp. cubense tropical race 4. - USDA-APHIS-PPQCPHST.

[26] Madhumitha, G., Rajakumar, G., Roopan, S. M., Rahuman, A. A., Priya, K. M., Saral, A. M., Khan, F. R. N., Khanna, V. G., Velayutham, K., Jayaseelan, C., Kamaraj, C., Elango, G. (2012): Acaricidal, insecticidal, and larvicidal efficacy of fruit peel aqueous extract of Annona squamosa and its compounds against blood-feeding parasites. - Parasitol Res 111: 2189-2199. doi:10.1007/s00436-011-2671-2.

[27] Manimegalai, S., Adhithya, R., Vellaikumar, S., Paramasivam, M., Chandrasekaran, S. (2011): Separation and characterization of antibacterial compounds from Aegle marmelos Correa and Thuja orientalis L. against silkworm pathogens. - International Journal of Genetic Engineering and Biotechnology 2(3): 251-260. http://www.irphouse.com.

[28] McChesney, J. D., Clark, A. M., Silveira, E. R. (1991): Antimicrobial diterpenes of Croton sonderianus, 1. Hardwickiic and 3,4-secotrachylobanoic acids. - J Nat Prod 54: 1625-1633.

[29] Moawad, A., Amin, E. (2019): Comparative antioxidant activity and volatile oil composition of leaves and fruits of Thuja orientalis growing in Egypt. - Walailak J Sci. \& Tech 16(11): 823-830.

[30] Monteiro, F. P., Ferreira, L. C., Silva, J. L., Pacheco, L. P., Souza, P. E. (2013): Influence of plant extracts and essential oils against Panama disease (Fusarium oxysporum f. sp. cubense) in banana seedlings. - Journal of agricultural science (5)4: 63-74. doi:10.5539/jas.v5n4p63.

[31] Muhammad, A., Hussain, I., Khanzada, K. A., Kumar, L., Ali, M., Yasmin, T., Hyder, M. Z. (2017): Molecular characterization of Fusarium oxysporum f. sp. cubense (FOC) tropical race 4 causing Panama disease in Cavendish banana in Pakistan. - Pak. J. Agri. Sci. 54(1). doi: https: //doi. org/10. 21162/ PAKJAS/17.4674.

[32] Mujeeb, F., Bajpai, P., Pathak, N. (2014): Phytochemical evaluation, antimicrobial activity, and determination of bioactive componenets from leaves of Aegle marmelos. - Biomed Research International.

[33] Naikwadi, S., Sannapapamma, K. J., Venugopal, C. K. (2017): Optimization of Vetiver Root Extract for Textile Finishing. - Int J Curr Microbiol App Sci 6(10): 2009-2022. doi: https:// doi.org/ 10.20546/ijcmas. 2017.610.238.

[34] Nascimento, G. G. F., Locatelli, J., Freitas, P. C., Silva, G. L. (2000): Antibacterial activity of plant extracts and phytochemicals on antibiotic-resistant bacteria. - Brazilian Journal of Microbiology 31: 247-256.

[35] Nene, Y., Thapilyal, L. (2000): Poisoned food technique of fungicides in plant disease control. $-3^{\text {rd }}$ ed. Oxford and IBH publishing company, New Delhi. 
[36] Ortiz, R., Swennen, R. (2014): From crossbreeding to biotechnology-facilitated improvement of banana and plantain. - Biotechnol Adv 32(1): 158-169. doi: 10.1016/j. biotechadv. 2013.09.010.

[37] Pegg, K. G., Coates, L. M., O’Neill, W. T., Turner, D. W. (2019): The Epidemiology of Fusarium Wilt of Banana. - Front Plant Sci 10: 1395. doi: 10.3389/fpls.2019.01395.

[38] Perrier, X., Langhe, E. D., Donohue, M., Lentfer, C., Vrydaghs, L., Bakry, F., Carreel, F., Hippolyte, I., Horry, J. P., Jenny, C., Lebot, V., Risterucci, A. M., Tomekpe, K., Doutrelepont, H., Ball, T., Manwaring, J., Maret, P. D., Denham, T. (2011): Multidisciplinary perspectives on banana (Musa spp.) domestication. - Proc Natl Acad Sci USA. 108: 11311-11318. doi: 10.1073/pnas.1102001108.

[39] Ploetz, R. C. (2000): Panama disease: A classic and destructive disease of banana. - Plant Health Progress. doi: 10.1094/PHP-2000-1204-01-HM.

[40] Ploetz, R. C. (2006): Fusarium wilt of banana is caused by several pathogens referred to as Fusarium oxysporum f. sp. cubense. - Phytopathology 96: 653-656. doi: 10.1094/PHYTO96-0653.

[41] Ploetz, R. C., Kepler, A. K., Daniells, J., Nelson, S. C. (2007): Banana and plantain-an overview with emphasis on Pacific Island cultivars. - In: Elevitch, C. R. (ed.) Species profile for Pacific Island agroforestry. Permanent Agriculture Resources (PAR), Holualoa, Hawaii, pp. 1-27.

[42] Ploetz, R. C. (2015): Fusarium wilt of banana. - Phytopathology 105(12): 1512-1521.

[43] Saravanakumar, D., Karthiba, L., Ramjegathesh, R., Prabakar, K., Raguchander, T. (2015): Characterization of bioactive compounds from botanicals for the management of plant diseases. - In: Ganesan, S., Vadivel, K., Jayaraman, J. (eds.) Sustainable crop disease management using natural products. CAB International.

[44] Sati, S. C., Joshi, S. (2010): Antibacterial potential of leaf extracts of Juniperus communis L. from Kumaun Himalaya. - African Journal of Microbiology Research 4(12): 1291-1294.

[45] Sharma, A., Joshi, S., Kumar, N. (2015): Antioxidant and antibacterial properties of leaves of Elaeocarpus sphaericus Roxb. and Pinus wallichiana from Uttarakhand region of India. - International Journal of Green Pharmacy 9(4): 246.

[46] Sharma, A., Sharma, L., Goyal, R. (2018): A Review on Himalayan Pine Species: Ethnopharmacological, Phytochemical and Pharmacological Aspects. - Pharmacogn J 10(4): 611-619. doi: 10. 5530/pj. 2018.4.100.

[47] Simmonds, N. W. (1986): Classification and breeding of bananas. - In: Persley, G. J., Langhe, E. A. D. (eds.) Banana and plantain breeding strategies. Proceedings of an international workshop, Cairns, Australia. 13-17 October, 1986. ACIAR proceedings 21, pp. 69-73.

[48] Siripornvisal, S. (2010): Antifungal activity of Ajowan oil against Fusarium oxysporum. KMITL Sci Tech J 10(2).

[49] Soylu, E. M., Soylu, S., Kurt, S. (2006): Antimicrobial activities of the essential oils of various plants against tomato late blight disease agent Phytophthora infestans. Mycopathologia 161: 119-128.

[50] Stover, R. H. (1962): Fusarial wilt (Panama disease) of bananas and other Musa species. CMI, Kew, Surrey, UK.

[51] Syed, R. N., Lodhi, A. M., Jiskani, M. M., Rajput, K. I., Khaskheli, M. A., Khanzada, M. A., Rajput, N. A., Maitlo, S. A., Rajput, A. Q. (2015): First report of Panama wilt disease of banana caused by Fusarium oxysporum f. sp. cubense in Pakistan. - Journal of Plant Pathology 97(1): 209-220. doi: http: //dx.doi. org/10.4454/JPP. V97I1.044.

[52] Vicente, L. P., Dita, M. A., Martínez, D. L. P. E. (2014): Prevention and diagnostic of Fusarium Wilt (Panama disease) of banana caused by Fusarium oxysporum f. sp. cubense tropical race 4 (TR4). - Technical manual prepared for the regional training workshop on the diagnosis of Fusarium Wilt organized by FAO regional office of the Caribbean and CARDI on 5-9 May in St. Augustine, Trinidad and Tobago, 74p. http:// www. fao.org/ 
fileadmin/ templates/ agphome/ documents/ Pests_Pesticides/ caribbeantr4/13 Manual Fusarium.pdf.

[53] Wink, M., Ashour, M. L., El-Readi, M. Z. (2012): Secondary metabolites from plants inhibiting $\mathrm{ABC}$ transporters and reversing resistance of cancer cells and microbes to cytotoxic and antimicrobial agents. - Front Microbiol 3: 1-15.

[54] Yin, X. M., Jin, Z. Q., Xu, B. Y., Ma, W. H., Fu, Y. G., Wang, J. B. (2011): Characterization of early events in banana root infected with the GFP-tagged Fusarium oxysporum f. sp. cubense. - Acta Horticulture 897: 371-376.

[55] Zhang, H., Mallik, A., Zeng, R. S. (2013): Control of Panama disease of banana by rotating and intercropping with Chineese Chive (Allium tuberosum Rottler): Role of plant volatiles. - J Chem Ecol. doi: 10.1007/s10886-013-0243-x.

\section{APPENDIX}

\section{Supplementary Information}

Table S1. Analysis of variance (factorial 3-way) applied for percent inhibition of Foc mycelia growth recorded for $5 \%$ and $10 \%$ of gymnosperm extracts prepared in $n$-hexane, ethanol and methanol solvents $(p<0.05)$

\begin{tabular}{c|c|c|c|c|c}
\hline Source & DF & SS & MS & F & P \\
\hline Replicate & 4 & 10 & 2.4 & & \\
Treatment & 12 & 84953 & 7079.5 & 956.93 & 0.0000 \\
Solvent & 2 & 3019 & 1509.4 & 204.03 & 0.0000 \\
Conc & 1 & 12178 & 12178.4 & 1646.15 & 0.0000 \\
Treatment*Solvent & 24 & 32780 & 1365.8 & 184.62 & 0.0000 \\
Treatment*Conc & 12 & 1235 & 102.9 & 13.91 & 0.0000 \\
Solvent*Conc & 2 & 200 & 99.8 & 13.48 & 0.0000 \\
Treatment*Solvent*Conc & 24 & 831 & 34.6 & 4.68 & 0.0000 \\
Error & 308 & 2279 & 7.4 & & \\
Total & 389 & 137485 & & & \\
\hline
\end{tabular}

Grand Mean 39.656, CV 6.86

Table S2. Pairwise comparisons test (Treatment*Solvent *Concentration) of percent inhibition of Foc mycelia growth recorded for 5\% and 10\% of gymnosperm extracts prepared in n-hexane, ethanol and methanol solvents

\begin{tabular}{c|c|c|c|c}
\hline Treatment & Solvent & Conc & Mean & Homogeneous Groups \\
\hline 8 & 3 & 2 & 66.666 & $\mathrm{~A}$ \\
9 & 1 & 2 & 66.664 & $\mathrm{~A}$ \\
7 & 1 & 2 & 66.074 & $\mathrm{AB}$ \\
11 & 1 & 2 & 66.070 & $\mathrm{AB}$ \\
5 & 2 & 2 & 65.832 & $\mathrm{AB}$ \\
11 & 3 & 2 & 65.474 & $\mathrm{ABC}$ \\
13 & 1 & 2 & 62.974 & $\mathrm{BCD}$ \\
4 & 2 & 2 & 62.384 & $\mathrm{CD}$ \\
5 & 1 & 2 & 60.592 & $\mathrm{DE}$ \\
7 & 2 & 2 & 60.474 & $\mathrm{DE}$ \\
8 & 2 & 2 & 60.116 & $\mathrm{DEF}$ \\
4 & 1 & 2 & 59.642 & $\mathrm{DEFG}$ \\
7 & 1 & 1 & 58.808 & $\mathrm{EFGH}$ \\
11 & 2 & 2 & 57.738 & $\mathrm{EFGH}$ \\
7 & 3 & 2 & 57.736 & $\mathrm{EFGH}$ \\
13 & 3 & 2 & 56.902 & FGHI \\
11 & 1 & 1 & 56.546 & GHIJ \\
9 & 1 & 1 & 56.306 & GHIJ \\
\hline
\end{tabular}




\begin{tabular}{|c|c|c|c|c|}
\hline Treatment & Solvent & Conc & Mean & Homogeneous Groups \\
\hline 5 & 3 & 2 & 56.068 & HIJ \\
\hline 5 & 1 & 1 & 55.952 & HIJ \\
\hline 3 & 3 & 2 & 54.172 & IJK \\
\hline 8 & 3 & 1 & 53.338 & JKL \\
\hline 13 & 1 & 1 & 52.138 & KLM \\
\hline 11 & 3 & 1 & 51.784 & KLM \\
\hline 12 & 1 & 2 & 51.780 & KLM \\
\hline 3 & 1 & 2 & 51.428 & KLM \\
\hline 5 & 2 & 1 & 50.238 & LMN \\
\hline 2 & 3 & 2 & 49.644 & MNO \\
\hline 8 & 2 & 1 & 49.166 & MNOP \\
\hline 7 & 2 & 1 & 48.810 & MNOP \\
\hline 4 & 2 & 1 & 47.736 & NOPQ \\
\hline 4 & 1 & 1 & 46.902 & NOPQR \\
\hline 7 & 3 & 1 & 46.308 & OPQRS \\
\hline 13 & 3 & 1 & 46.308 & OPQRS \\
\hline 4 & 3 & 2 & 46.078 & PQRS \\
\hline 9 & 3 & 2 & 44.642 & QRST \\
\hline 5 & 3 & 1 & 44.166 & RSTU \\
\hline 3 & 2 & 2 & 43.094 & STUV \\
\hline 12 & 1 & 1 & 42.260 & TUVW \\
\hline 11 & 2 & 1 & 42.020 & TUVW \\
\hline 3 & 3 & 1 & 41.186 & UVWX \\
\hline 12 & 3 & 2 & 40.474 & VWX \\
\hline 2 & 2 & 2 & 39.404 & WX \\
\hline 6 & 3 & 2 & 39.168 & WX \\
\hline 4 & 3 & 1 & 38.448 & $X Y$ \\
\hline 6 & 2 & 2 & 38.212 & XYZ \\
\hline 10 & 2 & 2 & 35.240 & $\mathrm{YZa}$ \\
\hline 12 & 2 & 2 & 34.880 & $\mathrm{Za}$ \\
\hline 9 & 2 & 2 & 34.764 & $\mathrm{a}$ \\
\hline 10 & 1 & 2 & 34.404 & $\mathrm{a}$ \\
\hline 9 & 3 & 1 & 33.928 & $\mathrm{a}$ \\
\hline 2 & 3 & 1 & 33.808 & $\mathrm{a}$ \\
\hline 2 & 1 & 2 & 33.692 & $a b$ \\
\hline 3 & 1 & 1 & 32.260 & $a b c$ \\
\hline 6 & 3 & 1 & 30.354 & bcd \\
\hline 10 & 3 & 2 & 30.354 & bcd \\
\hline 3 & 2 & 1 & 28.926 & cde \\
\hline 6 & 1 & 2 & 28.810 & de \\
\hline 2 & 1 & 1 & 27.498 & def \\
\hline 10 & 1 & 1 & 26.188 & efg \\
\hline 12 & 2 & 1 & 26.188 & efg \\
\hline 8 & 1 & 2 & 25.834 & efgh \\
\hline 10 & 3 & 1 & 24.284 & fghi \\
\hline 2 & 2 & 1 & 23.452 & ghi \\
\hline 6 & 2 & 1 & 22.498 & hij \\
\hline 10 & 2 & 1 & 20.952 & $\mathrm{ijk}$ \\
\hline 12 & 3 & 1 & 19.762 & $\mathrm{jkl}$ \\
\hline 9 & 2 & 1 & 18.808 & $\mathrm{kl}$ \\
\hline 8 & 1 & 1 & 17.260 & 1 \\
\hline 13 & 2 & 2 & 17.022 & 1 \\
\hline 6 & 1 & 1 & 12.498 & $\mathrm{~m}$ \\
\hline 13 & 2 & 1 & 1.5480 & $\mathrm{n}$ \\
\hline 1 & 1 & 1 & 0.0000 & $\mathrm{n}$ \\
\hline 1 & 1 & 2 & 0.0000 & $\mathrm{n}$ \\
\hline 1 & 2 & 1 & 0.0000 & $\mathrm{n}$ \\
\hline 1 & 2 & 2 & 0.0000 & $\mathrm{n}$ \\
\hline 1 & 3 & 1 & 0.0000 & $\mathrm{n}$ \\
\hline 1 & 3 & 2 & 0.0000 & $\mathrm{n}$ \\
\hline
\end{tabular}

Alpha 0.05 Standard Error for Comparison 1.7202, Critical T Value 1.968, Critical Value for Comparison 3.3849, Error term used: Replicate*TREATMENT*Solvent*Conc., $308 \mathrm{DF}$, there are 40 groups (A, B, etc.) in which the means are not significantly different from one another 
Table S3. Completely randomized analysis of variance for percent inhibition of Foc mycelia growth using $50 \%$ concentrations of the selected solvent extracts $(p<0.05)$

\begin{tabular}{c|c|c|c|c|c}
\hline Source & DF & SS & MS & F & P \\
\hline Treatment & 16 & 97956.4 & 6122.28 & 532 & 0.0000 \\
Error & 68 & 781.9 & 11.50 & & \\
Total & 84 & 98738.4 & & & \\
\hline
\end{tabular}

Grand Mean 57.387, CV 5.91

Table S4. Completely randomized analysis of variance for severity scoring of banana plants treated with different solvent extracts in green house experiment $(p<0.05)$

\begin{tabular}{c|c|c|c|c|c}
\hline Source & DF & SS & MS & F & P \\
\hline Treatment & 16 & 39.6471 & 2.47794 & 21.1 & 0.0000 \\
Error & 34 & 4.0000 & 0.11765 & & \\
Total & 50 & 43.6471 & & & \\
\hline
\end{tabular}

Grand Mean 3.6471, CV 9.40

Table S5. Completely randomized analysis of variance for measurement of banana pseudostem length measured in different treatments during greenhouse experiment $(p<0.05)$

\begin{tabular}{c|c|c|c|c|c}
\hline Source & DF & SS & MS & F & P \\
\hline Treatment & 16 & 1085.16 & 67.8225 & 13. & 0.0000 \\
Error & 34 & 167.47 & 4.9257 & & \\
Total & 50 & 1252.63 & & & \\
\hline
\end{tabular}

Grand Mean 35.030, CV 6.34

Table S6. Completely randomized analysis of variance for leaf width of banana plants measured in different treatments during greenhouse experiment $(p<0.05)$

\begin{tabular}{c|c|c|c|c|c}
\hline Source & DF & SS & MS & F & P \\
\hline Treatment & 16 & 192.539 & 12.0337 & 8.65 & 0.0000 \\
Error & 34 & 47.312 & 1.3915 & & \\
Total & 50 & 239.850 & & & \\
\hline
\end{tabular}

Grand Mean 15.006, CV 7.86

Table S7. Completely randomized analysis of variance for leaf length of banana plants measured in different treatments during greenhouse experiment $(p<0.05)$

\begin{tabular}{c|c|c|c|c|c}
\hline Source & DF & SS & MS & F & P \\
\hline Treatment & 16 & 619.498 & 38.7186 & 5.35 & 0.0000 \\
Error & 34 & 246.141 & 7.2394 & & \\
Total & 50 & 865.639 & & & \\
\hline
\end{tabular}

Grand Mean 31.429, CV 8.56 